\title{
Viscous tilting and production of vorticity in homogeneous turbulence
}

\author{
M. Holzner, ${ }^{1}$ M. Guala, ${ }^{2}$ B. Lüthi, ${ }^{1}$ A. Liberzon, ${ }^{3}$ N. Nikitin, ${ }^{4}$ W. Kinzelbach, ${ }^{1}$ \\ and A. Tsinober ${ }^{3}$ \\ ${ }_{1}^{1}$ Institute of Environmental Engineering, ETH Zurich, CH 8093 Zurich, Switzerland \\ ${ }^{2}$ Galcit, California Institute of Technology, Pasadena, California 91125, USA \\ ${ }^{3}$ School of Mechanical Engineering, Tel Aviv University, Ramat Aviv 69978, Israel \\ ${ }^{4}$ Institute of Mechanics, Moscow State University, 119899 Moscow, Russia
}

(Received 9 February 2010; accepted 9 May 2010; published online 8 June 2010)

\begin{abstract}
Viscous depletion of vorticity is an essential and well known property of turbulent flows, balancing, in the mean, the net vorticity production associated with the vortex stretching mechanism. In this letter, we, however, demonstrate that viscous effects are not restricted to a mere destruction process, but play a more complex role in vorticity dynamics that is as important as vortex stretching. Based on the results from three dimensional particle tracking velocimetry experiments and direct numerical simulation of homogeneous and quasi-isotropic turbulence, we show that the viscous term in the vorticity equation can also locally induce production of vorticity and changes of the orientation of the vorticity vector (viscous tilting). (c) 2010 American Institute of Physics.
\end{abstract}

[doi:10.1063/1.3442477]

In turbulent flows, the energy is injected at large scales by some forcing mechanism and dissipated into heat through the effect of viscosity at the smallest scales of motion, e.g., Ref. 1. The main physical mechanisms that control fluid turbulence at the smallest scales are commonly described in terms of strain and vorticity, quantities that represent the tendency of fluid parcels to deform and rotate, respectively.

One of the most prominent processes occurring at small scales is the so-called "vortex stretching:" following a common argument, ${ }^{1}$ if a vortical fluid element is stretched by the surrounding flow, the rotation rate should increase to conserve angular momentum. However, Lüthi et $a l^{2}{ }^{2}$ showed that this does not hold true pointwise and the dynamics is significantly influenced by a viscous contribution. The enstrophy balance equation

$$
\frac{D}{D t} \frac{\omega^{2}}{2}=\omega_{i} \omega_{j} s_{i j}+\nu \omega_{i} \nabla^{2} \omega_{i}
$$

where the squared vorticity magnitude $\omega^{2}$ denotes the enstrophy, $s_{i j}$ the rate of strain tensor, and $\nu$ the kinematic viscosity of the fluid, contains a production term $\omega_{i} \omega_{j} s_{i j}$ and a viscous term $\nu \omega_{i} \nabla^{2} \omega_{i}$. The two terms in the mean (hereinafter mean values $\langle\cdot\rangle$ are obtained by spatial and temporal averaging) approximately balance each other, i.e., $\left\langle\omega_{i} \omega_{j} s_{i j}\right\rangle$ $\simeq-\left\langle\nu \omega_{i} \nabla^{2} \omega_{i}\right\rangle$, see Ref. 1 . The presence of a viscous contribution in Eq. (1) shows that the effect of molecular viscosity is not limited to energy dissipation through deformation work, expressed as $\varepsilon=2 \nu s_{i j} s_{i j}$, but, among other things, it controls also vorticity growth. The effects of vortex stretching and viscous destruction are usually captured in the wellknown picture that in turbulence at small scales the nonlinearities increase gradients, whereas the viscosity depletes them, e.g., Refs. 1 and 3 and references therein. However, as noted already by, e.g., Tennekes and Lumley, ${ }^{1}$ viscous effects are not restricted to vorticity destruction only. For example, viscosity may tilt vorticity, see, e.g., Refs. 1 and $3-6$, and is believed to be responsible for vortex reconnection, e.g., Refs. $3-5$. It is reminded that this "classical" reconnection mechanism (due to viscosity) is fundamentally different from reconnection events in quantum fluids, which take place due to a quantum stress acting at the scale of the vortex core without changes of total energy. ${ }^{7,8}$ However, direct experimental evidence for the occurrence of tilting and production of vorticity due to viscosity is still missing in the literature, also because up to now it is difficult to measure the associated small scale quantities experimentally. Derivatives of the velocity became accessible through particle tracking experiments since the developments in, e.g., Refs. 2, 9, and 10. Holzner et al. ${ }^{10}$ recently measured the viscous production of vorticity, $\nu \omega_{i} \nabla^{2} \omega_{i}>0$, in the proximity of turbulent/ nonturbulent interfaces, which raised the question about the role of positive $\nu \omega_{i} \nabla^{2} \omega_{i}$ in fully developed and homogeneous turbulence.

In this letter we present the first measurements of tilting, depletion, and considerable production of vorticity through viscosity in a turbulent flow using particle tracking velocimetry (PTV) (Refs. 2, 9, and 10). The main goal is to unfold viscous effects on vorticity dynamics at the small scales of turbulence with emphasis on genuine (i.e., intrinsic to Navier-Stokes turbulence as opposed to kinematic) effects. The results discussed hereafter are based on higher order derivatives and are challenging to obtain, both experimentally and numerically, which is why we compare the experimental results with those obtained through direct numerical simulation (DNS).

We measured the flow velocity and its gradients in a laboratory experiment of homogeneous, quasi-isotropic, and statistically stationary turbulence by using PTV, see Refs. 2 and 11 for details. PTV is based on high speed imaging of the motion of small buoyant tracer particles seeded into the flow. The experiment was carried out in a glass tank filled with water and the flow was forced mechanically from two 


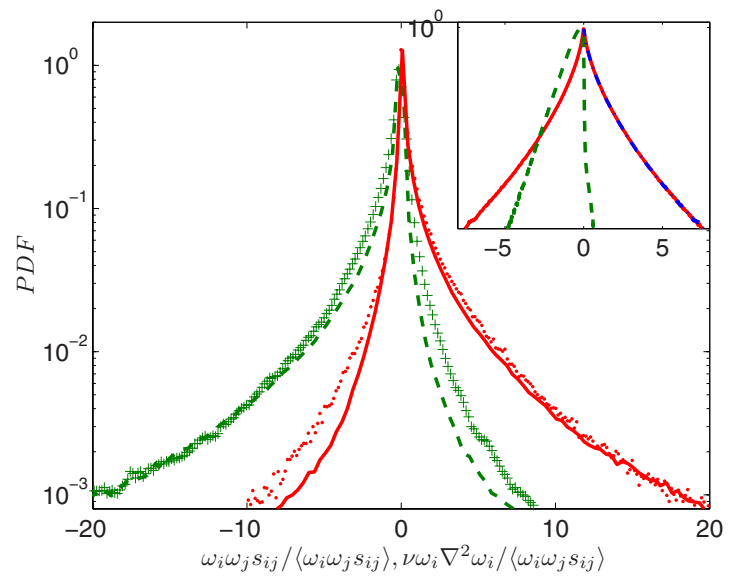

FIG. 1. (Color online) PDFs of $\omega_{i} \omega_{j} s_{i j}(-, \mathbf{O})$ and $\nu \omega_{i} \nabla^{2} \omega_{i}(--,+)$ normalized with $\left\langle\omega_{i} \omega_{j} s_{i j}\right\rangle$. Symbols are from PTV, lines from DNS. The inset shows the analogous results from a random Gaussian velocity field, $\omega_{i} \omega_{j} s_{i j}$ $(-), \omega_{i} \nabla^{2} \omega_{i}(--)$, the vertical reflection of the PDF corresponding to negative events, $\omega_{i} \omega_{j} s_{i j}<0(-\cdot-)$, demonstrates the symmetry.

sides by two sets of rotating disks as in Ref. 11 . The observation volume of approximately $15 \times 15 \times 20 \mathrm{~mm}^{3}$ was centered with respect to the forced flow domain, midway between the disks. The turbulent flow is characterized by a rms velocity of about $10 \mathrm{~mm} / \mathrm{s}$, a Taylor-based Reynolds number of $\operatorname{Re}_{\lambda}=50$, and the Kolmogorov length and time scales are estimated at $\eta=0.5 \mathrm{~mm}$ and $\tau_{\eta}=0.25 \mathrm{~s}$, respectively. The Laplacian of vorticity $\nabla^{2} \boldsymbol{\omega}$ is obtained indirectly from the local balance equation of vorticity in the form $\nabla \times \mathbf{a}=\nu \nabla^{2} \boldsymbol{\omega}$ by evaluating the term $\nabla \times \mathbf{a}$ from the particle tracking data, where $\mathbf{a}=D \mathbf{u} / D t$ is the Lagrangian acceleration. Through this indirect method only one derivative in space is needed instead of three, but particle positions have to be differentiated twice in time in order to get the Lagrangian acceleration. For the numerical simulation we used an open source turbulence database that was developed at Johns Hopkins University, see Ref. 12 for details. The data are from a DNS of forced isotropic turbulence on a $1024^{3}$ periodic grid using a pseudospectral parallel code. The TaylorReynolds number is $\operatorname{Re}_{\lambda}=434$. After the simulation had reached a statistically stationary state, 1024 frames of data, which include the three components of the velocity vector and pressure, were generated and stored into the database. The time interval covered by the numerical data set is thus only one large-eddy turnover time, whereas it is $O(10)$ turnover times for the experiment. For comparison to a random velocity field, divergence-free Gaussian white noise was generated as in Ref. 13.

First, we statistically analyze the effects of viscosity on the vorticity magnitude. One of the most basic phenomena of three dimensional turbulence is the predominant vortex stretching, which is manifested in a positive net enstrophy production, $\left\langle\omega_{i} \omega_{j} s_{i j}\right\rangle>0$, e.g., Refs. 1 and 3 and references therein. A strong positive skewness of the probability density function (PDF) of the term $\omega_{i} \omega_{j} s_{i j}$ is indeed visible in Fig. 1, in agreement with earlier results, e.g., Ref. 3. For statistically stationary turbulence the growth of enstrophy is balanced by viscous effects, i.e., the two terms on the right hand side of
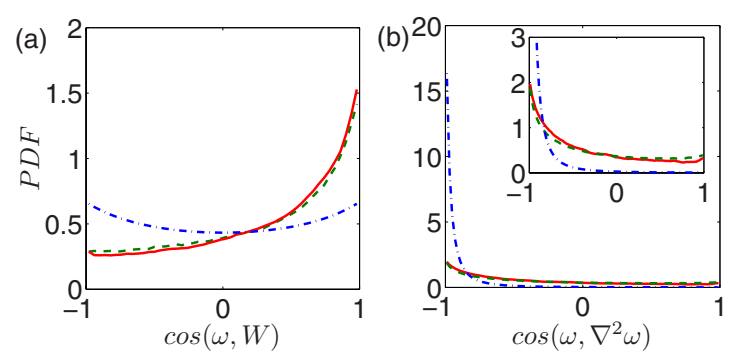

FIG. 2. (Color online) PDFs of the cosine of the angle (a) between vorticity and the vortex stretching vector and (b) between vorticity and its Laplacian, as obtained from DNS (-), PTV (- $)$, and a random Gaussian field (-- -).

Eq. (1) balance in the mean. Consistently, the term $\nu \omega_{i} \nabla^{2} \omega_{i}$ shows an opposite distribution, being strongly negatively skewed (Fig. 1). Although viscosity mostly depletes enstrophy, we note that also events where $\nu \omega_{i} \nabla^{2} \omega_{i}>0$ are statistically significant. In fact, about one third of all events represent viscous production of enstrophy. The experimental curves qualitatively agree with the numerical ones, the PDFs obtained from DNS are slightly more skewed. It is important to note that, while the reasons for the positiveness of the mean enstrophy production term are dynamical and due to interaction between vorticity and strain, the destructive nature of the viscous term $\left\langle\nu \omega_{i} \nabla^{2} \omega_{i}\right\rangle<0$ arises also for kinematical reasons: the term $\nu \omega_{i} \nabla^{2} \omega_{i}$ can be decomposed into two contributions (note that this is one of many possibilities, i.e., it is not a unique decomposition), e.g.,

$$
\omega_{i} \nabla^{2} \omega_{i}=\nabla \cdot[\omega \times(\nabla \times \omega)]-(\nabla \times \omega)^{2},
$$

where the first term on the right hand side is a divergence of a vector and vanishes in the mean for homogeneity, whereas the second is a (always negative) dissipation term. ${ }^{14}$ Indeed, while for a Gaussian random field the PDF of $\omega_{i} \omega_{j} s_{i j}$ becomes symmetric and $\left\langle\omega_{i} \omega_{j} s_{i j}\right\rangle$ is vanishing identically, ${ }^{15}$ the PDF of the viscous term is strongly negatively skewed, see the inset in Fig. 1. This means that the destructive nature of the viscous term is also recovered in a random field and does not represent a genuine property of turbulent flow fields. However, from the same inset, we estimate that for a random Gaussian field, the events with $\omega_{i} \nabla^{2} \omega_{i}>0$ are statistically far less significant (about 2\% of all events) compared to the same events in a Navier-Stokes field (about 30\%). We, therefore, conclude that considerable viscous production of vorticity is a genuine characteristic of Navier-Stokes turbulence.

The positiveness of the mean enstrophy production is associated with the predominant alignment between vorticity and the vortex stretching vector. The enstrophy production can be expressed as the scalar product of vorticity and the vortex stretching vector, $\omega_{i} \omega_{j} s_{i j}=\boldsymbol{\omega} \cdot \mathbf{W}$, where $W_{i}=\omega_{j} s_{i j}$. In real turbulent flows, the two vectors are strongly aligned. Thus, the PDF of the cosine of the angle between $\boldsymbol{\omega}$ and $\mathbf{W}$ is asymmetric [Fig. 2(a)], in conformity with the prevalence of vortex stretching over vortex compression, whereas it is symmetric for a random Gaussian field [Fig. 2(a)], see also Ref. 3 and references therein. A value of $\cos (\cdot)=-1$ represents "antialignment" or antiparallel vectors, $\cos (\cdot)=0$ 


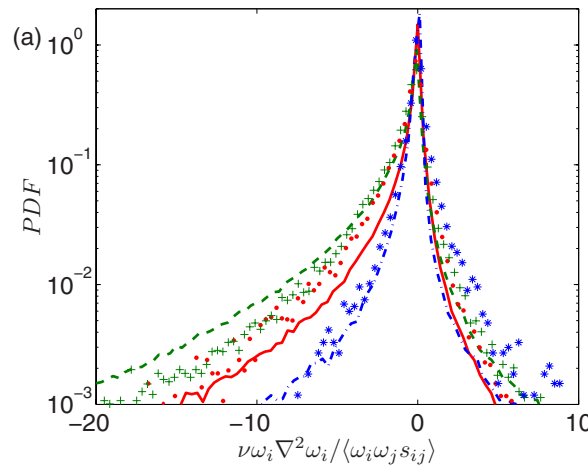

(b)

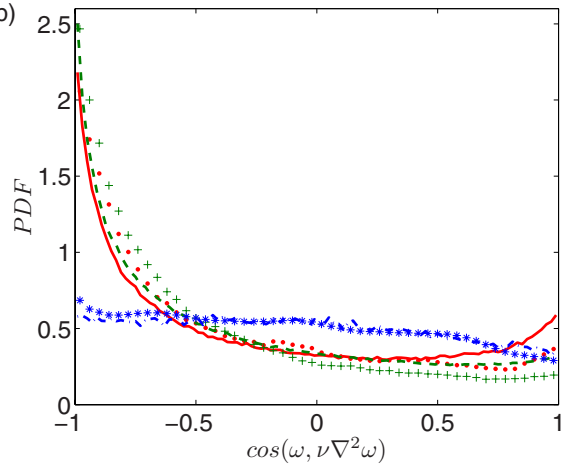

FIG. 3. (Color online) PDFs of $\nu \omega_{i} \nabla^{2} \omega_{i}$ (a) and of the cosine of the angle between vorticity and its Laplacian (b) for different $\left(\boldsymbol{\omega}-\boldsymbol{\lambda}_{i}\right)$ alignments from DNS (lines) and PTV (symbols), $\boldsymbol{\omega}$ aligned with $\boldsymbol{\lambda}_{\mathbf{1}}(-, \boldsymbol{\bullet}), \boldsymbol{\lambda}_{\mathbf{2}}(--,+)$, and $\boldsymbol{\lambda}_{3}(-\cdot-, *)$.

represents "no alignment" or perpendicular vectors, and $\cos (\cdot)=1$ represents "aligned" or parallel vectors. Analogously, we show the alignment between $\boldsymbol{\omega}$ and $\nabla^{2} \boldsymbol{\omega}$ in Fig. 2(b). The figure shows high probabilities (much higher for the random field) of pronounced antialignment between $\boldsymbol{\omega}$ and $\nabla^{2} \boldsymbol{\omega}$, consistent with the negative skewness of the PDF of $\nu \omega_{i} \nabla^{2} \omega_{i}$, but we also note that with some smaller probability the two vectors can attain any orientation and, in particular, they can also be strongly aligned. This reminds of the results in Ref. 10 , which measured $\cos \left(\boldsymbol{\omega}, \nabla^{2} \boldsymbol{\omega}\right) \simeq 1$ in the proximity of the interface between turbulent and irrotational flow regions. The fact that the two vectors are not always strictly antialigned implies that the term $\nu \nabla^{2} \boldsymbol{\omega}$ does not act exclusively in the direction of the vorticity vector (mostly dampening and sometimes increasing the vorticity magnitude), but also normally to it, thus contributing to altering the orientation of vorticity. Since the negative skewness of the PDF is much stronger for the random velocity field than for the turbulent one, we may infer that viscous tilting is characteristic of fluid turbulence. The observation that the viscous term can effectively influence the orientation of vorticity is important, also because this will affect the relative orientation between $\boldsymbol{\omega}$ and $\boldsymbol{\lambda}_{i}$ and therefore indirectly influence the vortex stretching (compression) mechanism.

The inviscid tilting of vorticity was measured by Guala et $a{ }^{6}{ }^{6}$ and found to be sensitive to the alignments between vorticity and the strain eigenvectors. With the present data it is possible to estimate for the first time both the inviscid and the viscous contributions to the tilting of vorticity and to quantify the influence of the relative $\left(\boldsymbol{\omega}-\boldsymbol{\lambda}_{\boldsymbol{i}}\right)$ alignments. We adopt the approach of Ref. 6 and condition the data on situ-

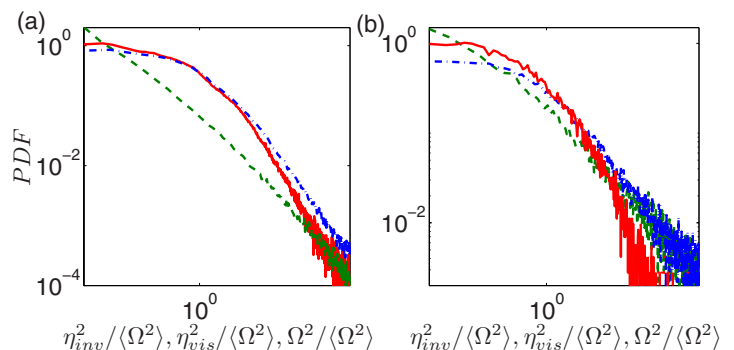

FIG. 4. (Color online) PDFs of inviscid (-), viscous $(--)$, and total (---) tilting from DNS (left) and PTV (right).

ations of different alignments of vorticity with the principal axis of the strain eigenframe. Note that in a Gaussian field no differences are observed when conditioning on such alignments and therefore the expected effects in turbulent flow are explicitly dynamical.

Figure 3(a) depicts the PDFs of the viscous term divided into the three subsets depending on the local alignment between $\boldsymbol{\omega}$ and $\boldsymbol{\lambda}_{i}$. The subsets are divided according to the condition $\cos ^{2}\left(\boldsymbol{\omega}, \boldsymbol{\lambda}_{i}\right) \geq 0.7$, corresponding to a cone of roughly $33^{\circ}$, as in Ref. 6 . It is visible that, while for the case of alignment with the intermediate eigenvector $\boldsymbol{\lambda}_{2}$, the PDF becomes more skewed, i.e., $\nu \omega_{i} \nabla^{2} \omega_{i}$ contributes more to the reduction of $\omega^{2}$, in the case of alignment with $\boldsymbol{\lambda}_{\mathbf{1}}$, the skewness decreases and even more so when vorticity is aligned with $\boldsymbol{\lambda}_{\mathbf{3}}$. Again, the main qualitative trends are the same both for the numerical and experimental results, with the curves obtained from DNS showing a stronger skewness.

In Fig. 3(b) we analyze how this qualitatively different behavior of the term $\nu \omega_{i} \nabla^{2} \omega_{i}$ is reflected in the alignment between $\boldsymbol{\omega}$ and $\nabla^{2} \boldsymbol{\omega}$. The PDF of the cosine of the angle between the two vectors is strongly negatively skewed for the cases when $\boldsymbol{\omega}$ is aligned with $\boldsymbol{\lambda}_{\mathbf{1}}$ and $\boldsymbol{\lambda}_{\mathbf{2}}$. In the case of $\boldsymbol{\omega}$ aligned with $\boldsymbol{\lambda}_{\mathbf{3}}$ the distribution changes dramatically becoming very flat in conformity with the reduced skewness of the PDF of $\nu \omega_{i} \nabla^{2} \omega_{i}$. Therefore, in this case viscosity contributes less to the destruction of enstrophy, but still plays a role, e.g., for the tilting of the vorticity vector.

The inviscid and the viscous contributions to the total tilting $\boldsymbol{\Omega}$ of vorticity can be written as follows:

$$
\Omega_{k}=\frac{D \hat{\omega}_{k}}{D t}=\eta_{\omega_{k}}^{i}+\eta_{\omega_{k}}^{v}
$$

where

$$
\eta_{\omega_{k}}^{i}=\frac{\omega_{j} s_{k j}}{\omega}-\frac{\omega_{l} \omega_{j} s_{l j}}{\omega^{3}} \omega_{k}
$$

and

$$
\eta_{\omega_{k}}^{v}=\frac{\nu \nabla^{2} \omega_{k}}{\omega}-\frac{\nu \omega_{j} \nabla^{2} \omega_{j}}{\omega^{3}} \omega_{k}
$$

represent the inviscid and viscous tilting, respectively, and $\hat{\omega}_{k}=\omega_{k} /|\boldsymbol{\omega}|$ is the vorticity unit vector. Figure 4 shows PDFs of the squared magnitudes of total, inviscid, and viscous tilting and it appears that viscous tilting is typically smaller than the inviscid one, but at large magnitudes both contributions to the total tilting are comparably significant. The PDFs of 
viscous and total tilting obtained from PTV appear to be somewhat higher at the tails compared to the numerical result, but the experimental scatter is considerable at high magnitudes. In order to appreciate the dependence of the tilting magnitudes on geometrical properties introduced before, it is useful to write the following equations:

$$
\begin{aligned}
\left(\eta_{\omega}^{i}\right)^{2} & =\sqrt{\mathbf{W}^{2} / \omega^{2}} \sin ^{2}(\boldsymbol{\omega}, \mathbf{W}) \\
& =\Lambda_{k}^{2} \cos ^{2}\left(\boldsymbol{\omega}, \boldsymbol{\lambda}_{k}\right)-\left[\Lambda_{k} \cos ^{2}\left(\boldsymbol{\omega}, \boldsymbol{\lambda}_{k}\right)\right]^{2},
\end{aligned}
$$

and

$$
\left(\eta_{\omega}^{v}\right)^{2}=\sqrt{\left(\nu \nabla^{2} \boldsymbol{\omega}\right)^{2} / \omega^{2}} \sin ^{2}\left(\boldsymbol{\omega}, \nabla^{2} \boldsymbol{\omega}\right) .
$$

From Eq. (5) one can see that the inviscid tilting vanishes identically when $\boldsymbol{\omega}$ is strictly aligned with $\boldsymbol{\lambda}_{i}$. This alignment can then only be changed in two ways: through viscous tilting and/or through a change of the orientation of the strain eigenframe.

In summary, in this letter we have shown that viscosity in two thirds of all events depletes enstrophy and that there is an essential contribution of kinematic nature to this effect. Viscous tilting and production of vorticity, which occur in one third of all events, are instead characteristic features of turbulent flows. Our results demonstrate that viscosity influences enstrophy production by changing the vorticity in magnitude and direction. The observed effects are sensitive to the $\left(\boldsymbol{\omega}-\boldsymbol{\lambda}_{i}\right)$ alignments and thus to the local vortex stretching (compression) regime. When $\boldsymbol{\omega}$ is aligned with $\boldsymbol{\lambda}_{\mathbf{3}}$ the purely destructive contribution of $\nu \omega_{i} \nabla^{2} \omega_{i}$ is strongly suppressed. From the technical point we note that the experimental and numerical results agree well with each other on the qualitative level. Some quantitative discrepancies might be attributed to the fact that experimental measurements are affected by limited spatial resolution and noise and to the difference in Reynolds numbers. In the future it might be possible to repeat the analysis and possibly verify the observed trends for much higher Reynolds numbers. Finally, we propose a plausible postulate regarding the role of viscosity for the predominant $\boldsymbol{\omega}-\boldsymbol{\lambda}_{\mathbf{2}}$ alignment so typical for turbulent flows, e.g., Refs. 16 and 17. In these situations the vectors $\boldsymbol{\omega}$ and $\nabla^{2} \boldsymbol{\omega}$ are predominantly antialigned. Strong $\boldsymbol{\omega}-\boldsymbol{\lambda}_{2}$ alignment stalls inviscid tilting, while the antialignment of $\boldsymbol{\omega}$ and $\nabla^{2} \boldsymbol{\omega}$ points to reduced viscous tilting, i.e., both mechanisms could work toward maintaining the alignment. In future work we hope to pursue these questions that are intimately related to moderation of enstrophy growth and to prevention of finite time singularities. ${ }^{18}$ This will also require to address the tilting mechanisms of the strain eigenframe. The work presented here, together with the related recent work on velocity gradient dynamics in turbulent flow, e.g., Ref. 19, comprises a challenge for any closure based on local information, e.g., Ref. 20, and generally for a more complete comprehension of the velocity gradient dynamics.

${ }^{1}$ H. Tennekes and J. L. Lumley, A First Course in Turbulence (MIT, Cambridge, MA, 1972).

${ }^{2}$ B. Lüthi, A. Tsinober, and W. Kinzelbach, "Lagrangian measurement of vorticity dynamics in turbulent flow," J. Fluid Mech. 528, 87 (2005).

${ }^{3}$ A. Tsinober, An Informal Conceptual Introduction to Turbulence (Springer, New York, 2009).

${ }^{4}$ S. Kida and M. Takaoka, "Breakdown of frozen motion of vorticity field and vorticity reconnection," J. Phys. Soc. Jpn. 60, 2184 (1991).

${ }^{5}$ S. Kida and M. Takaoka, "Vortex reconnection," Annu. Rev. Fluid Mech. 26, 169 (1994).

${ }^{6}$ M. Guala, B. Lüthi, A. Liberzon, W. Kinzelbach, and A. Tsinober, "On the evolution of material lines and vorticity in homogeneous turbulence," J. Fluid Mech. 533, 339 (2005).

${ }^{7}$ M. Leadbeater, T. Winiecki, D. C. Samuels, C. F. Barenghi, and C. S. Adams, "Sound emission due to superfluid vortex reconnections," Phys. Rev. Lett. 86, 1410 (2001).

${ }^{8}$ M. S. Paoletti, M. E. Fisher, K. R. Sreenivasan, and D. P. Lathrop, "Velocity statistics distinguish quantum turbulence from classical turbulence," Phys. Rev. Lett. 101, 154501 (2008).

${ }^{9}$ G. A. Voth, A. LaPorta, A. M. Crawford, J. Alexander, and E. Bodenschatz, "Measurement of particle accelerations in fully developed turbulence," J. Fluid Mech. 469, 121 (2002).

${ }^{10}$ M. Holzner, A. Liberzon, N. Nikitin, W. Kinzelbach, and A. Tsinober, "Small-scale aspects of flows in proximity of the turbulent/nonturbulent interface," Phys. Fluids 19, 071702 (2007).

${ }^{11}$ K. Hoyer, M. Holzner, B. Lüthi, M. Guala, A. Liberzon, and W. Kinzelbach, "3D scanning particle tracking velocimetry," Exp. Fluids 39, 923 (2005).

${ }^{12}$ Y. Li, E. Perlman, M. Wan, Y. Yang, C. Meneveau, R. Burns, S. Chen, A. Szalay, and G. Eyink, "A public turbulence database cluster and applications to study Lagrangian evolution of velocity increments in turbulence," J. Turbul. 9, 31 (2008).

${ }^{13}$ W. J. T. Bos, L. Liechtenstein, and K. Schneider, "Small-scale intermittency in anisotropic turbulence," Phys. Rev. E 76, 046310 (2007).

${ }^{14}$ It is noteworthy that the above decomposition of $\nu \omega_{i} \nabla^{2} \omega_{i}$-although useful-has a limitation since it is not unique and there is an infinite number of possibilities to represent it as a sum of a dissipation and a flux term (i.e., as a divergence of some vector), e.g., $\nu \omega_{i} \nabla^{2} \omega_{i}$ $=\nu \nabla^{2}\left(\omega^{2} / 2\right)-\nu \nabla \omega_{i}: \nabla \omega_{i}$. There is no way to define dissipation (i.e., to choose one among many purely negative expressions) of enstrophy as it is not an inviscidly conserved quantity, unlike the kinetic energy (Ref. 3).

${ }^{15}$ L. Shtilman, M. Spector, and A. Tsinober, "On some kinematic versus dynamic properties of homogeneous turbulence," J. Fluid Mech. 247, 65 (1993).

${ }^{16}$ W. T. Ashurst, A. R. Kerstein, R. M. Kerr, and C. H. Gibson, "Alignment of vorticity and scalar gradient with strain rate in simulated Navier-Stokes turbulence," Phys. Fluids 30, 2343 (1987).

${ }^{17}$ P. E. Hamlington, J. Schumacher, and W. J. A. Dahm, "Local and nonlocal strain rate fields and vorticity alignment in turbulent flows," Phys. Rev. E 77, 026303 (2008).

${ }^{18}$ R. M. Kerr and A. Brandenburg, "Evidence for a singularity in ideal magnetohydrodynamics: Implications for fast reconnection," Phys. Rev. Lett. 83, 1155 (1999).

${ }^{19} \mathrm{~B}$. Lüthi, M. Holzner, and A. Tsinober, "Expanding the $Q-R$ space to three dimensions," J. Fluid Mech. 641, 497 (2009).

${ }^{20}$ L. Chevillard, C. Meneveau, L. Biferale, and A. Toschi, "Modeling the pressure Hessian and viscous Laplacian in turbulence: Comparisons with direct numerical simulation and implications on velocity gradient dynamics," Phys. Fluids 20, 101504 (2008) 